\title{
The Dynamic Performance Analysis of a Low-Floor Tram Hydraulic Anti-Kink System Based on Multidisciplinary Collaboration
}

\author{
Xiaokang Liao ${ }^{1}$, Zili Chen ${ }^{2, *}$, Yiping Jia ${ }^{3}$ and Jianhui Lin ${ }^{4}$ \\ 1 School of Electrical Engineering, Southwest Jiaotong University, Chengdu 611730, Sichuan, China; \\ lxklsxxh1218@163.com \\ 2 School of Mathematics, Southwest Jiaotong University, Chengdu 611730, Sichuan, China \\ 3 Sichuan Aerospace Industry Group Co., Ltd., Chengdu 610100, Sichuan, China; 13541055077@163.com \\ 4 State Key Laboratory of Traction Power, Southwest Jiaotong University, Chengdu 610036, Sichuan, China; \\ lin13008104673@126.com \\ * Correspondence: zlchen@swjtu.edu.cn; Tel.: +86-139-8005-0975
}

Received: 5 August 2020; Accepted: 18 August 2020; Published: 21 August 2020

check for updates

\begin{abstract}
According to the basic principle of the hydraulic anti-kink system and flow continuity equation, this paper takes the low-floor tram as the research object and the four vehicles as the research carrier. Based on the correlation parameters between the vehicle subsystem and the hydraulic subsystem, a co-simulation platform of a low-floor tram with hydraulic an anti-kink system is built. The co-simulation results show that the anti-kink system can well maintain the relative yaw angle consistency between the vehicle body and bogie. The anti-kink system restrains the maximum yaw angle and excessive lateral displacement of the vehicle body effectively. The consistency between the experiment results and the simulation results shows the accuracy of the model. The co-simulation model of the low-floor tram with hydraulic anti-kink system can be used to research the dynamic performance when it passes through curve line.
\end{abstract}

Keywords: hydraulic anti-kink system; low-floor tram; co-simulation; dynamic performance

\section{Introduction}

With the continuous expansion of the city boundary, the traffic problems inside the city are becoming increasingly serious. As an efficient means of transportation, urban rail transit has been paid more and more attention by urban decision makers. Among them, the low-floor tram has been widely used in many cities because of its economic and environmental protection, energy saving, and other characteristics [1,2]. The low-floor tram is composed of three motor vehicles Mc1, M, Mc2, and a trailer T. The overall structure of a low-floor tram is shown in Figure 1. Mc1 and T form a unit and M and Mc2 form another unit. The two units are connected by a single hinge joint, and only two vehicles are allowed to yaw around the hinge center in the horizontal plane [3]. Because this kind of tram adopts the structure of one bogie for each vehicle, the yaw angle of the vehicle body relative to the bogie in the plane is larger when crossing the curve. When the train passes through the curve, the bogie and articulated device will bear a certain torque, which can cause the relative rotation of the vehicle body and the lateral force on the wheel flange to increase. The excessive torque may even cause the train derailment. The installation of a hydraulic anti-kink system can restrain the yaw angle of the vehicle body relative to the bogie and greatly reduce the lateral force on the wheel flange [4]. Therefore, it is essential to research the curving performance and running stability of the low-floor tram. 


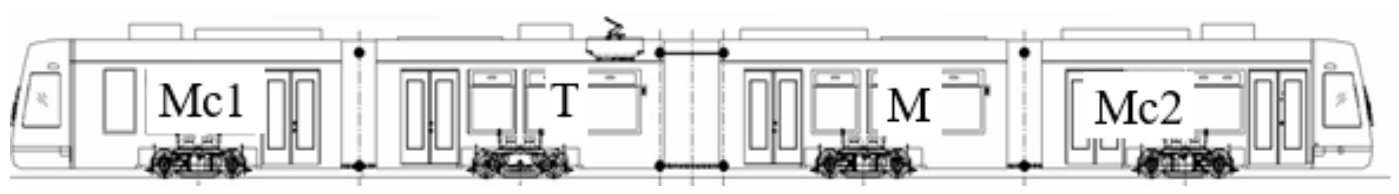

Figure 1. Overall structure of low-floor tram.

Uhl T. et al. [5] researched the structural dynamic characteristics of the low-floor tram by experimental method, including the strain measurement at the hinge joint and some motion devices in the vehicle body. They used a non-contact sensor to measure the displacement, yaw angle, and some mutual characteristics describing vehicle dynamics. Zhang X. et al. [6] established the dynamic model of a low-floor tram, determined the inner and outer widening amount of low-floor tram when running on the curve. They analyzed the dynamic performance index of low-floor tram with or without an anti-kink system. However, their analysis lacked the state quantity calculation of the hydraulic system, which had certain limitations. According to the flow continuity equation and force balance equation, Huang Y.P. et al. [7] established the dynamic model of the hydraulic anti-kink system of the tram and analyzed the dynamic response characteristics of the hydraulic anti-kink system. Their results showed that the force of the hydraulic cylinder controlled by the anti-kink system is different, and the force of the hydraulic cylinder near the guide wheel of the front vehicle is the largest. Zhu W.L et al. [8] established the models of the control subsystem, air brake subsystem, electric brake subsystem, and brake execution subsystem by using multidisciplinary collaborative analysis method. Their results provided theoretical basis for the development and design optimization of brake system. Li J. et al. [9] established the damping mathematical model which affected the lateral damping function of the anti-kink system by analyzing the principle of the anti-kink system. They analyzed the influence of throttle valve and pressure limiting valve in the buffer valve group on the damping characteristics. The simulation curve of damping characteristics was in good agreement with the test curve. Wang Y.Q. et al. [10] designed two sets of the hydraulic anti-kink system, which can satisfy the operation state of anti-kink system in normal mode, fault mode, and anti-kink mode. They took the articulated Mc1 and vehicle T as their research objects. The two sets of anti-kink system were simulated under normal operation mode by AMESim software, they obtained the displacement of vehicle Mc1's main control cylinder and vehicle T's main control cylinder piston. Ding W.S. et al. [11] based on the establishment of a corresponding mathematical model and analyzed the formation principle of combined damping. The influence of the design parameters of the buffer valve group on the damping characteristics was further analyzed. Their results showed that the combination of throttle valve and pressure limiting valve in the buffer valve group forms the damping characteristics of multi segment parabola combination with multi inflection points. Liu X. et al. [12] established a lateral dynamic curve negotiation model of suspension rail vehicle bogies based on the coupling system of rubber tire and ground. They analyzed the lateral and yaw motion dynamics of wheel set structure with rubber tire and ground coupling, the displacement and acceleration response of bogie were calculated when vehicle frame and wheelset pass through curve section. Based on the theory of vehicle track coupling dynamics, Wang K.Y. et al. [13] simulated the performance indexes of wheel rail dynamic lateral interaction between locomotive and vehicle when passing through different curve tracks, including passenger and freight trains passing through mountain railway with a small radius curve, $160 \mathrm{~km} / \mathrm{h}$ passenger train and $200 \mathrm{~km} / \mathrm{h}$ freight train passing through curve track with different radius. Zboinski K. et al. [14] researched the nonlinear lateral stability of rail vehicles on the curve, and proposed a method to solve the more complex rail vehicle model.

Complex products have the intersection of multi-disciplinary information, and the manufacturing cycle of physical prototype is long and the cost is high. With the development of computer hardware and the technical progress in the field of single subject simulation, the product analysis of virtual prototype of complex products using multidisciplinary joint simulation technology plays an increasingly important role in the process of product development. The traditional empirical formula and estimation 
method are difficult to satisfy the design requirements of a low-floor tram when crossing the curve. The simulation method of multidisciplinary collaborative simulation is used to design and calculate such that a comparison is made between experiment and simulation. This is an effective way to realize the rational optimization design of low-floor tram. This paper took the low-floor tram as the research object and the four vehicles as the research carrier, based on the multidisciplinary collaborative analysis method and the correlation parameters among subsystems. The simulation platform of SIMPACK (A mechanical dynamics software) vehicle dynamic model, AMESim (A complex system modeling and simulation software) anti-kink system hydraulic model and Simulink model are built to research the influence of hydraulic anti-kink system on the dynamic performance of the low-floor tram under curve conditions.

\section{Model of Hydraulic Anti-Kink System}

\subsection{Structure of Anti-Kink System}

The hydraulic anti-kink system is installed between the vehicle body and the bogie. The hydraulic cylinder, elastic stop, and lateral shock absorber are used to restrain the yaw motion of the vehicle body. When the vehicle body moves laterally, the oil in the hydraulic pipeline flows in the high-pressure chamber and low-pressure chamber of the hydraulic cylinder. The hydraulic oil flows through the throttle valve to play a damping role. When the vehicle yaws its head, the hydraulic oil in the high-pressure chamber of the two hydraulic cylinders is controlled by the one-way valve, and flows to the same pipeline. The buffer hydraulic cylinder reduces the oil pressure in the hydraulic pipeline. When the front end of the vehicle enters the curve, the vehicle body yaws its head. Because of the buffer hydraulic cylinder, the pressure in the hydraulic pipeline does not rise sharply, but increase slowly. At this time, the anti- kink system allows the angle between the front and rear vehicle bodies and the corresponding bogies to have a certain difference, which plays the critical role of anti yaw damping. When the rear vehicle body enters the curve, the pressure maintained in the buffer cylinder pushes the rear body to make the head yaw and play the role of auxiliary steering. Therefore, the parameter setting of buffer hydraulic cylinder is the key of hydraulic anti-kink system. The simplified schematic diagram of hydraulic anti-kink system is shown in Figure 2.

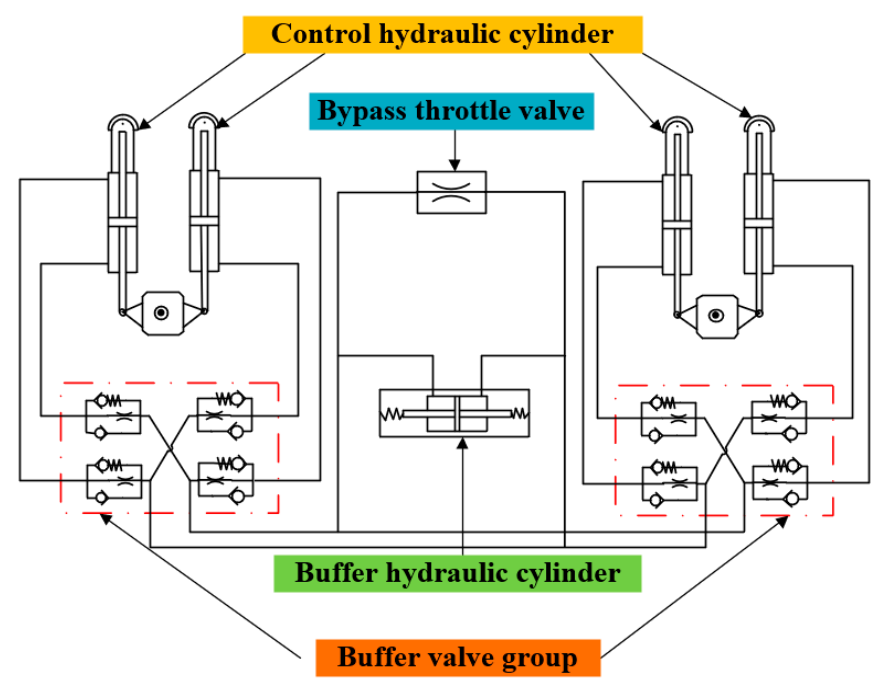

Figure 2. Simplified schematic diagram of hydraulic anti-kink system.

\subsection{Mathematical Model of Anti-Kink System}

The anti-kink system can keep the yaw angle of two vehicle bodies $\gamma_{1}$ and $\gamma_{2}$ in a unit equal to their respective bogies. When the two angles are not equal, the anti-kink system produces a reverse torque 
$M_{\gamma}$ between the bogie and the vehicle body, forcing the two angles to be consistent. The equation of torque and yaw angle is satisfied [15]:

$$
M_{\gamma 1}=-M_{\gamma 2}=K_{\gamma}\left(\gamma_{1}-\gamma_{2}\right)
$$

Here: $M_{\gamma 1}$ is the torque between the front vehicle body and its bogie, $M_{\gamma 2}$ is the torque between rear vehicle and its bogie, and $K_{\gamma}$ is the rotation stiffness between vehicle bodies.

When the two ends of the hydraulic cylinder are respectively connected with the vehicle body and the bogie, the relationship between the yaw angle $\gamma$ and the displacement $Y$ of the moving parts of the hydraulic cylinder is expressed as:

$$
Y=H \gamma
$$

Here: $H$ is the longitudinal distance from the connection point of the anti-kink system and the vehicle body to the center of the bogie.

In order to establish the mathematical model of the hydraulic anti-kink system composed of a control hydraulic cylinder, buffer hydraulic cylinder, and bypass throttle valve, the simplified model of the control hydraulic cylinder was analyzed. The simplified model of the hydraulic anti-kink system is shown in Figure 3.

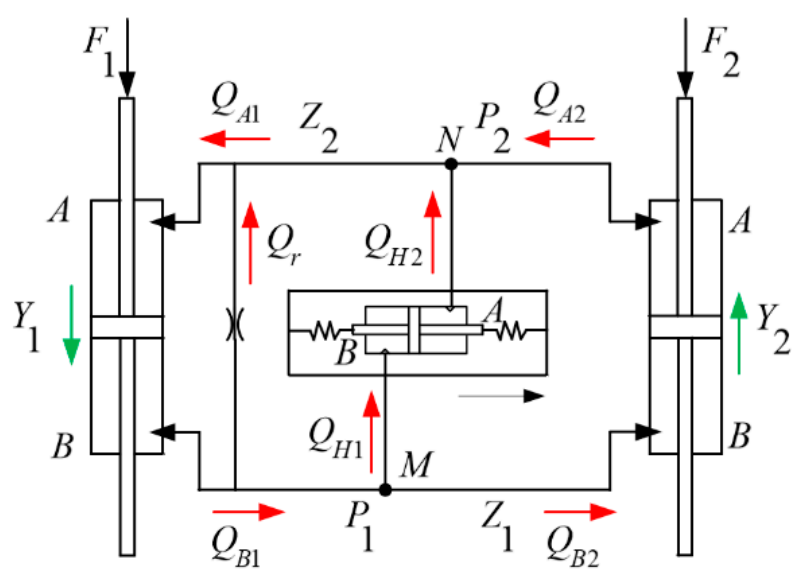

Figure 3. Simplified model of the hydraulic anti-kink system.

Assume that the piston sectional area and initial volume of each hydraulic cylinder are $A_{0}$ and $V_{0}$. The sectional area and initial volume of the combined hydraulic cylinder in Figure 3 are $2 A_{0}$ and $2 V_{0}$. Suppose that the control hydraulic cylinder 1 produces a downward displacement $Y_{1}$ under the action of $F_{1}$, and the control hydraulic cylinder 2 has an upward displacement $Y_{2}$ under the action of pressure, and the resistance is $F_{2}$. The flow continuity equation of control hydraulic cylinder 1 can be expressed as:

$$
\begin{aligned}
& 2 A_{0} \frac{d Y_{1}}{d t}-Q_{B 1}=\frac{2 V_{0}-2 A_{0} Y_{1}}{\beta_{e}} \frac{d P_{1}}{d t} \\
& Q_{A 1}-2 A_{0} \frac{d Y_{1}}{d t}=\frac{2 V_{0}+2 A_{0} Y_{1}}{\beta_{e}} \frac{d P_{2}}{d t}
\end{aligned}
$$

Here: $Q_{A 1}$ is the inlet oil flow of chamber $\mathrm{A}, Q_{B 1}$ is the flow of oil from chamber $\mathrm{B}, P_{1}$ is the pressure of control hydraulic cylinder chamber $\mathrm{B}, \mathrm{P}_{2}$ is the pressure of control hydraulic cylinder chamber $\mathrm{A}$, and $\beta_{e}$ is the bulk modulus of hydraulic oil.

The differential equation of the moving parts of hydraulic cylinder 1 is expressed as:

$$
M_{k} \frac{d^{2} Y_{1}}{d t^{2}}=F_{1}-\left(P_{1}-P_{2}\right) A_{0}-\delta \frac{d Y_{1}}{d t}
$$


Here: $M_{k}$ is the mass of the moving part of the hydraulic cylinder and $\delta$ is the motion damping coefficient of piston cylinder. According to the flow continuity formula, the flow equations of chamber $\mathrm{A}$ and chamber $\mathrm{B}$ of the buffer cylinder are expressed as:

$$
\begin{aligned}
& A_{H} \frac{d x}{d t}-Q_{H 2}=\frac{V_{1}-A_{H} x}{\beta_{e}} \frac{d P_{2}}{d t} \\
& Q_{H 1}-A_{H} \frac{d x}{d t}=\frac{V_{1}+A_{H} x}{\beta_{e}} \frac{d P_{1}}{d t}
\end{aligned}
$$

The differential equation of the moving parts of the buffer cylinder is expressed as:

$$
M_{H} \frac{d^{2} x}{d t^{2}}+\delta \frac{d x}{d t}+2 K_{t} x=A_{H}\left(P_{1}-P_{2}\right)
$$

Here: $M_{H}$ is the mass of the moving part of the buffer cylinder, $V_{1}$ is the initial volume of chamber A and chamber B of buffer hydraulic cylinder, and $K_{t}$ is the spring stiffness.

The flow continuity equation of control hydraulic cylinder 2 can be expressed as:

$$
\begin{aligned}
& 2 A_{0} \frac{d Y_{2}}{d t}-Q_{A 2}=\frac{2 V_{0}-2 A_{0} Y_{2}}{\beta_{e}} \frac{d P_{2}}{d t} \\
& Q_{B 2}-2 A_{0} \frac{d Y_{2}}{d t}=\frac{2 V_{0}+2 A_{0} Y_{2}}{\beta_{e}} \frac{d P_{1}}{d t}
\end{aligned}
$$

Here: $Q_{A 2}$ is the inlet oil flow of chamber $\mathrm{A}$ and $Q_{B 2}$ is the flow of oil from chamber B. The differential equation of the moving parts of hydraulic cylinder 2 is expressed as:

$$
M_{k} \frac{d^{2} Y_{2}}{d t^{2}}=\left(P_{1}-P_{2}\right) A_{0}-\delta \frac{d Y_{2}}{d t}-F_{2}
$$

The flow rate of the bypass throttle valve is expressed by the following formula:

$$
Q_{r}=C_{d} A_{2} \sqrt{\frac{2}{\rho}\left(P_{1}-P_{2}\right)}
$$

Here: $Q_{r}$ is the flow through the throttle valve, $C_{d}$ is the throttle flow coefficient, $\rho$ is the oil density, and $A_{2}$ is the orifice area of bypass throttle valve.

The flow continuity equation at node $\mathrm{M}$ is:

$$
Q_{B 1}=Q_{r}+Q_{H 1}+Q_{B 2}
$$

The flow continuity equation at node $\mathrm{N}$ is:

$$
Q_{A 1}=Q_{r}+Q_{H 2}+Q_{A 2}
$$

\subsection{AMESim Model of Anti-Kink System}

When the vehicle body yaws its head relative to the bogie, the oil flows through the buffer valve group into or out of the hydraulic cylinder, which plays the role of shock absorption. According to the basic principle of hydraulic anti-kink system, the internal hydraulic simulation model is constructed by AMESim software, as shown in Figure 4. 


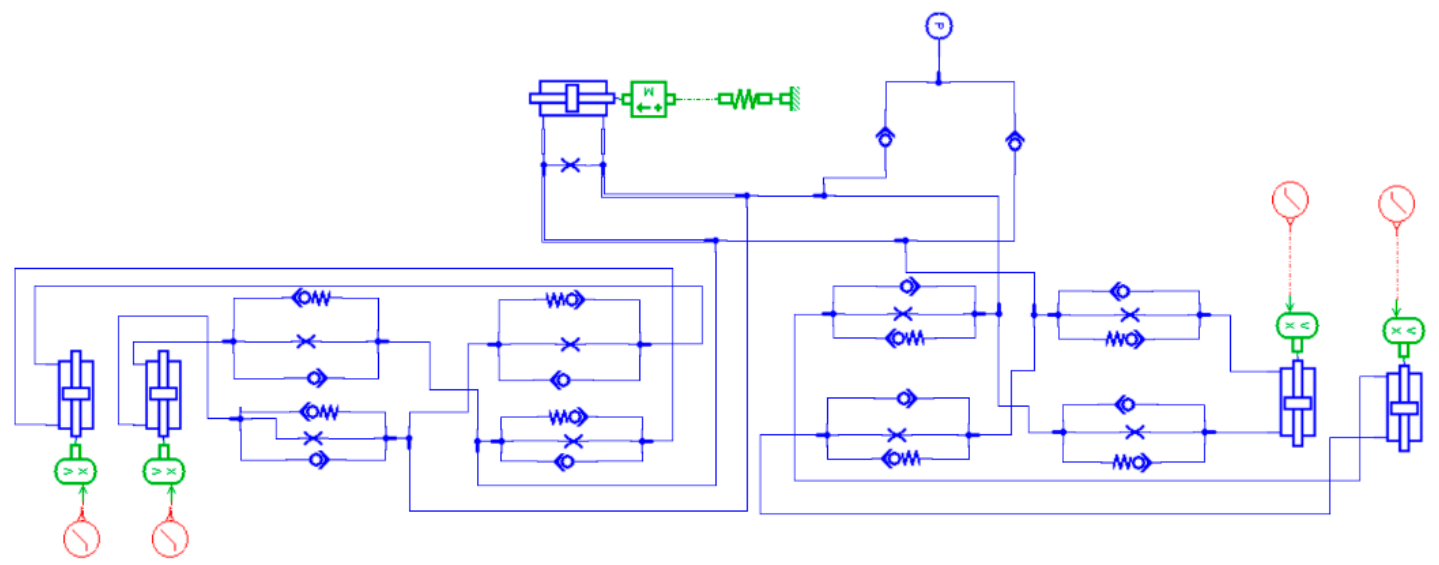

Figure 4. AMESim hydraulic simulation model.

The basic simulation parameters of anti-kink system are shown in Table 1.

Table 1. Basic simulation parameters of anti-kink system.

\begin{tabular}{cc}
\hline Name & Value \\
\hline Piston diameter of control cylinder $(\mathrm{mm})$ & 50 \\
Piston rod diameter of control cylinder $(\mathrm{mm})$ & 30 \\
Orifice area of throttle valve $\left(\mathrm{mm}^{2}\right)$ & 1.28 \\
Oil density $\left(\mathrm{kg} / \mathrm{m}^{3}\right)$ & 842 \\
Bulk modulus of oil $(\mathrm{GPa})$ & 1.46 \\
\hline
\end{tabular}

\section{SIMPACK Model of Low-Floor Tram}

The low-floor tram is different from the traditional railway vehicles. It uses the independent rotating wheel as the supporting motion device, and the four wheels are independent individuals. The front and rear wheels on both sides of the power bogie are coupled together through gears. The longitudinal coupling is simulated by the gear transmission force element in the whole vehicle modeling. Referring to Figure 1, the SIMPACK model of low-floor tram is shown in Figure 5. The position of each ball is the centroid of the corresponding vehicle body.

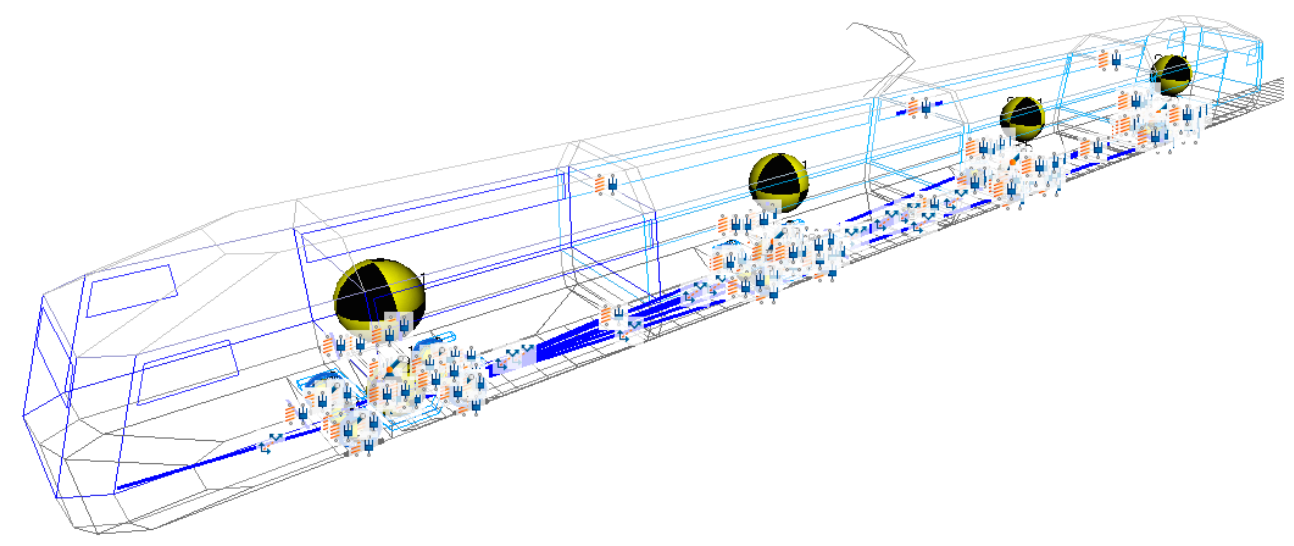

Figure 5. SIMPACK model of the low-floor tram.

The basic simulation parameters of the low-floor tram are shown in Table 2. 
Table 2. Basic simulation parameters of the low-floor tram.

\begin{tabular}{ccccc}
\hline Description & Mc1 & T & M & Mc2 \\
\hline Vehicle body mass (kg) & 10,221 & 9233 & 7793 & 9983 \\
Unspring mass per wheelset (kg) & 720 & 875 & 720 & 720 \\
Bogie mass (Not include unspring mass) $(\mathrm{kg})$ & 3430 & 1540 & 3420 & 3430 \\
Roll inertia of bogie (Not include unspring mass) $\left(\mathrm{kg} \cdot \mathrm{m}^{3}\right)$ & 2500 & 790 & 2500 & 2500 \\
Pitch inertia of bogie (Not include unspring mass) $\left(\mathrm{kg} \cdot \mathrm{m}^{3}\right)$ & 1000 & 500 & 1000 & 1000 \\
Yaw inertia of bogie (Not include unspring mass) $\left(\mathrm{kg} \cdot \mathrm{m}^{3}\right)$ & 3050 & 1200 & 3050 & 3050 \\
\hline
\end{tabular}

\section{The Co-Simulation of SIMPACK/AMESim/Simulink}

In order to better analyze the influence of the anti-kink system on vehicle driving performance under curve crossing conditions, the vehicle dynamic model based on SIMPACK and the hydraulic anti-kink system model based on AMESim are established. By defining the interaction interface between different software simulation models in Simulink environment, the integration of vehicle dynamics analysis simulation model and the anti-kink system hydraulic simulation model is realized. The scheme of the vehicle co-simulation platform is established as shown in Figure 6.

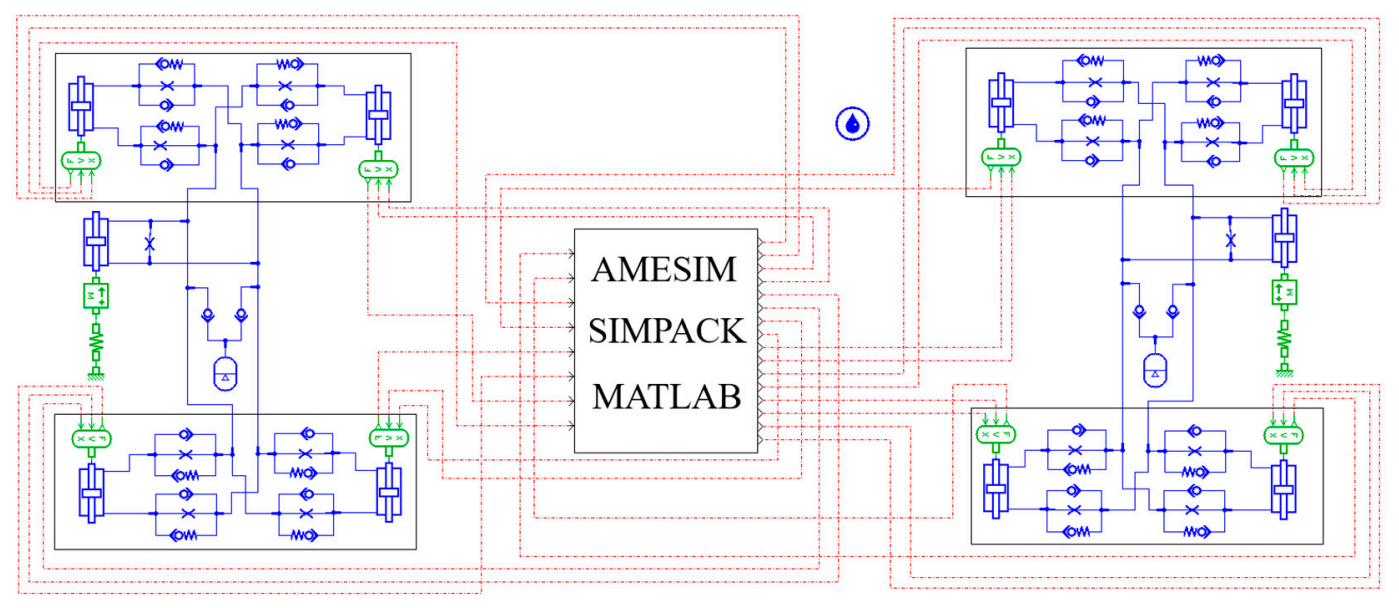

Figure 6. Scheme of the vehicle co-simulation platform.

\section{Influence of the Anti-Kink System on Vehicle Dynamic Performance}

In an urban rail transit system, the most common small curve basically includes S-shaped curve lines and C-shaped curve lines. Therefore, it is very important to research the performance of the hydraulic anti-kink system when the curve is too small. An S-shaped curve line and a C-shaped curve line are shown in Figure 7.
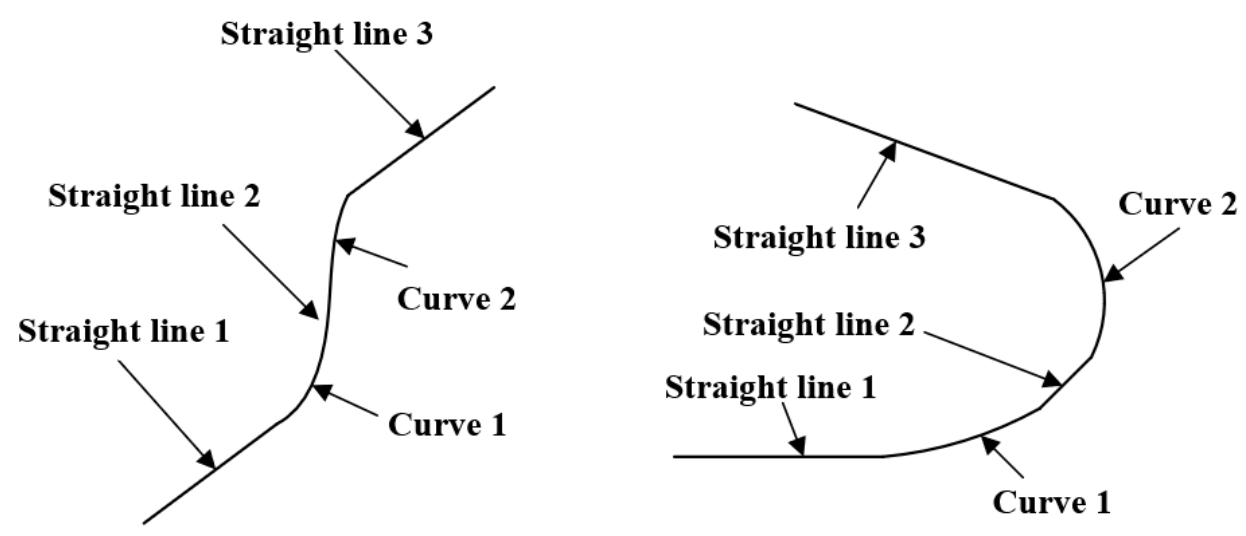

Figure 7. S-shaped curve line and C-shaped curve line. 
According to the requirements of code for design of railway lines (GB 50090-2006) and general technical conditions for low-floor tram vehicles (CJ/T 417-2012), the specific parameters of an S-shaped curve line and a $\mathrm{C}$-shaped curve line are as follows:

S-shaped curve line and C-shaped curve line: the length of straight line 1 and 3 is $50 \mathrm{~m}$, and the length of straight line 2 is $10 \mathrm{~m}$, the length of two circular curves is $10 \mathrm{~m}$ and the radius is $25 \mathrm{~m}$.

\subsection{Evaluation Index of Dynamic Performance}

The safety of vehicle operation mainly involves whether the vehicle will derail and overturn. Generally, derailment coefficient, wheel load reduction rate, wheel-rail lateral force and other indicators are used to evaluate the safety of vehicle operation. At present, China's vehicle departments mainly use derailment coefficient and wheel load reduction rate.

\subsubsection{Derailment Coefficient}

When the vehicle is running, the wheel derailment may be caused under the most unfavorable combination of line condition, operation condition, vehicle structural parameters, and loading. The "derailment coefficient" is used to evaluate the stability of wheel derailment, and the calculation formula is given by Nadal [16]:

$$
\frac{Q}{P}=\frac{\operatorname{tg} \alpha-\mu}{1+\mu \cdot \operatorname{tg} \alpha}
$$

Here: $Q$ is the lateral force acting on the wheel, $P$ is the vertical force acting on the wheel, $\mu$ is the friction coefficient at the flange, and $\alpha$ is the maximum flange contact angle.

The International Union of Railways (UIC) stipulates that: $Q / P \leq 1.2$.

\subsubsection{Wheel Unloading Rate}

The vertical force and lateral force of the wheel are the main parameters affecting derailment. Combined with the definition of the derailment coefficient, the main reason for derailment in theory is that the lateral force is too large. However, in practical application, it is found that sometimes the lateral force is small, but the derailment phenomenon may occur when the wheel load is reduced. Therefore, the wheel load reduction rate is used as a supplement to jointly determine the derailment safety of vehicles.

$$
\bar{W}=\frac{1}{2}\left(W_{1}+W_{2}\right) \quad \Delta W=\frac{1}{2}\left(W_{2}-W_{1}\right)
$$

Here: $\bar{W}$ is the average wheel weight of the unloaded side wheel and the loaded side wheel, $\Delta W$ is the load reduction variation of wheel load, $W_{1}$ is the wheel weight of the unloaded side wheel, and $W_{2}$ is the wheel weight of the loaded side wheel.

\subsubsection{Wheel-Rail Lateral Force}

Excessive wheel-rail lateral force will lead to deformation or even damage of the line, so the maximum value of lateral force should be controlled. According to GB5599-85, the maximum allowable value of wheel-rail lateral force during curve passing is shown in formula (17) and formula (18).

When the track spike is pulled out, the stress of the track spike is the limit of elastic limit:

$$
Q \leq 19+0.39 P_{s t}
$$

When the track spike is pulled out, the stress of the track spike is the limit of yield limit:

$$
Q \leq 29+0.39 P_{s t}
$$

Here: $Q$ is the lateral force acting on the wheel and $P_{s t}$ is the wheel static load. 
The wheel rail lateral force shall not exceed the design load of elastic fastener. The lateral force limit of Shinkansen in Japan and Europe and America is usually 0.4 times of axle load.

$$
Q \leq 0.4\left(P_{s t 1}+P_{s t 2}\right)
$$

Here: $P_{s t 1}$ is the static load of the left wheel and $P_{s t 2}$ is the static load of the right wheel.

\subsubsection{Vehicle Running Stability Index}

The evaluation indexes of vehicle running stability mainly include vehicle running stability index and comfort index. These indexes can not only reflect the performance of vehicle system, but also reflect the physical reaction of passengers to vehicle running quality.

This paper mainly introduces Sperling stability index, and evaluates vehicle running stability through this index. The formula of stationarity index $\mathbb{N}$ is shown as:

$$
\boldsymbol{\aleph}=2.7^{10} \sqrt{Z^{3} f^{5} F(f)}=0.896^{10} \sqrt{\frac{a^{3}}{f} F(f)}
$$

Here: $Z$ is the amplitude, $f$ is the vibration frequency, $a$ is the vibration acceleration, and $F(f)$ is the factor related to vibration frequency. The values of $F(f)$ are shown in Table 3.

Table 3. Frequency correction factor.

\begin{tabular}{cccc}
\hline \multicolumn{2}{c}{ Vertical Vibration } & \multicolumn{2}{c}{ Lateral Vibration } \\
\hline $0.5-5.9 \mathrm{~Hz}$ & $F(f)=0.325 f^{2}$ & $0.5-5.4 \mathrm{~Hz}$ & $F(f)=0.8 f^{2}$ \\
$5.9-20 \mathrm{~Hz}$ & $F(f)=400 / f^{2}$ & $5.4-26 \mathrm{~Hz}$ & $F(f)=650 / f^{2}$ \\
$>20 \mathrm{~Hz}$ & $F(f)=1$ & $>26 \mathrm{~Hz}$ & $F(f)=1$ \\
\hline
\end{tabular}

The stationarity index can only be applied to a single vibration of one frequency and one amplitude. But in the actual line, the variation of vibration frequency and amplitude with time is relatively complex. Therefore, before calculating the vehicle stability index, we analyze the frequency spectrum of the measured vehicle vibration acceleration to obtain the amplitude value of each frequency range, and then calculate the ride comfort index $\aleph_{t}$ of each band, and finally calculate the total stability index of all bands.

$$
\left(\aleph_{t}=\aleph_{1}{ }^{10}+\aleph_{2}{ }^{10}+\cdots+\aleph_{n}{ }^{10}\right)^{0.1}
$$

The vehicle stability levels specified in GB5599-85 are shown in Table 4. The running stability of ordinary passenger cars in China shall not be lower than the secondary standard, that is, the lateral and vertical stability indexes shall not exceed 2.75 .

Table 4. Passenger and Freight train stability level.

\begin{tabular}{cccc}
\hline \multirow{2}{*}{ Stability Level } & \multirow{2}{*}{ Evaluation Result } & \multicolumn{2}{c}{ Stability Index } \\
\cline { 3 - 4 } & & Passenger Train & Freight Train \\
\hline Class A & excellent & $<2.5$ & $<3.5$ \\
Class B & good & $2.5-2.75$ & $3.5-4.0$ \\
Class C & qualified & $2.75-3.0$ & $4.0-4.25$ \\
\hline
\end{tabular}

\subsection{S-Shaped Curve Line}

The change of angle between vehicle body and bogie with time for tram running on S-shaped curve line with the anti-kink system is shown in Figure 8. 


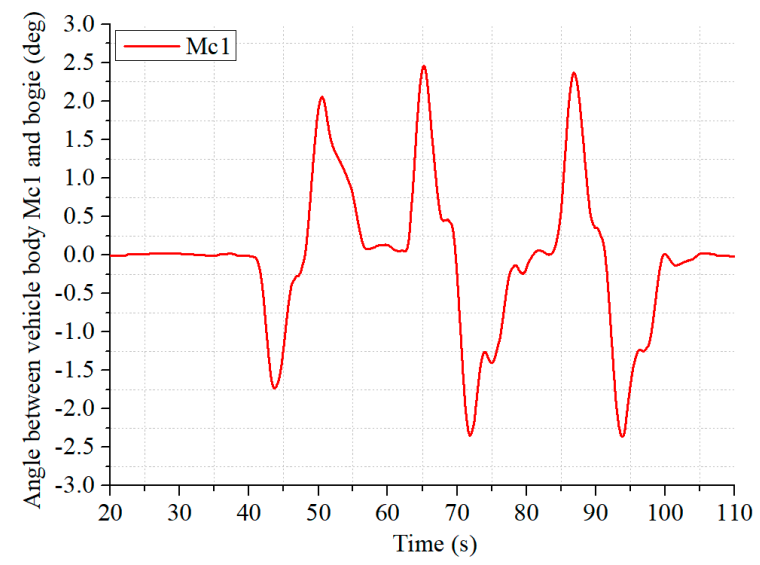

(A) Angle between vehicle body Mc1 and bogie

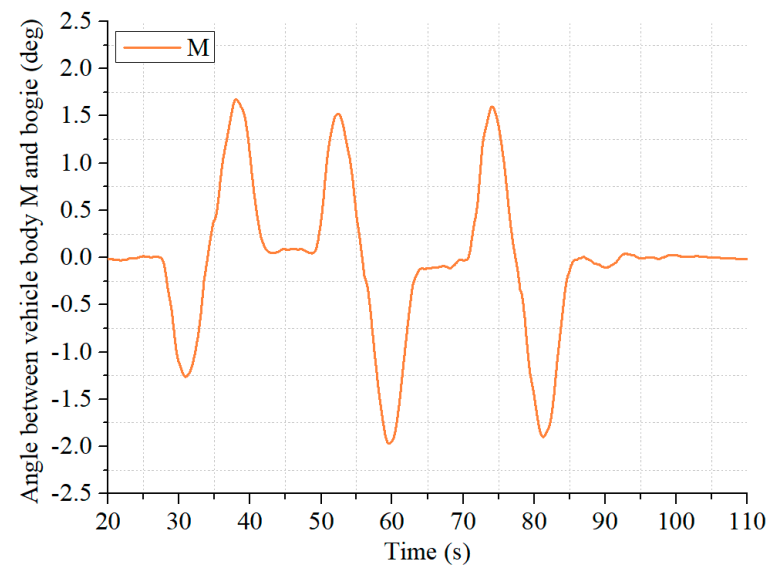

(C) Angle between vehicle body $\mathrm{M}$ and bogie

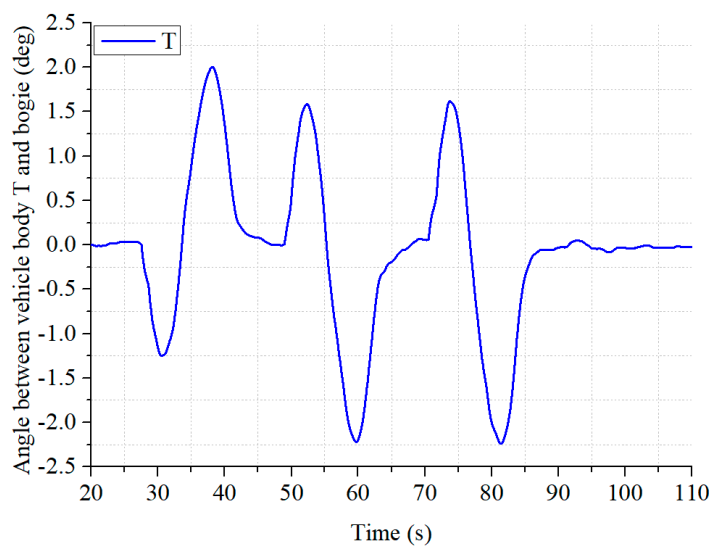

(B) Angle between vehicle body $\mathrm{T}$ and bogie

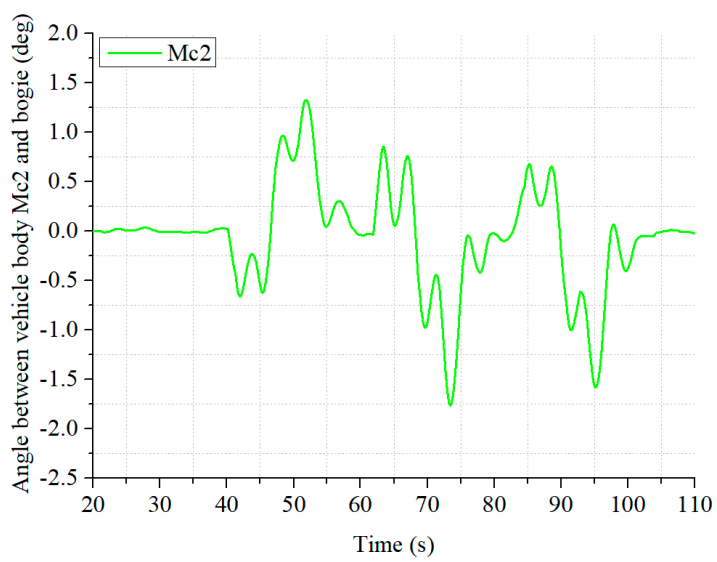

(D) Angle between vehicle body Mc2 and bogie

Figure 8. Angle between vehicle and bogie with hydraulic anti-kink system.

When the vehicle with the anti-kink system passes through the S-shaped curve, the change trend of the angle between the vehicle body and its bogie is different. The maximum angle between the vehicle body and the bogie can reach $2.42^{\circ}$, which is located in the Mc1 vehicle. Due to the buffer hydraulic cylinder of the working hydraulic anti-kink system, the overall stiffness is relatively small, allowing a certain difference in the angle between the front and rear vehicle bodies and the corresponding bogies. There will be a certain difference between the angles between the vehicle body and the bogie, and there is a delay in the rotation of the front and rear vehicle bodies relative to the corresponding bogies.

Figure 9 shows the change of the maximum derailment coefficient of each wheel group under the operating conditions. Before and after the installation of anti-kink system, the derailment coefficient of most wheel groups changes slightly, while the derailment coefficient of the two front wheel groups changes greatly. When the vehicle passes through the S-shaped curve at a faster speed, the most front-end bogie suffers a greater lateral impact. The maximum derailment coefficient without anti-kink system reaches 1.45 , the maximum derailment coefficient of installing anti-kink system wheel set is 0.72 . Figure 10 shows the change of wear index. After the anti-kink system is installed, the change range of wear index is small, the wear of front wheel set is large, and the wear of rear wheel set is small. Figure 11 shows the variation of the maximum wheel unloading rate under operating conditions. The maximum wheel unloading rate of the vehicles without the anti-kink system reaches 0.57 , close to the specified limit, and the maximum wheel unloading rate with the anti-kink system is 0.28 . Figure 12 shows the variation of the maximum wheel-rail lateral force under the operating conditions. The maximum 
wheel-rail lateral force of the vehicles without the anti-kink system reaches $82.8 \mathrm{KN}$, far exceeding the maximum value allowed by the standard. The maximum wheel-rail transverse force of the vehicles with anti-kink system is $38.2 \mathrm{KN}$, which is lower than the national standard value of $43 \mathrm{KN}$. Therefore, the anti-kink system can improve the safety of a single tram passing through the S-shaped curve.

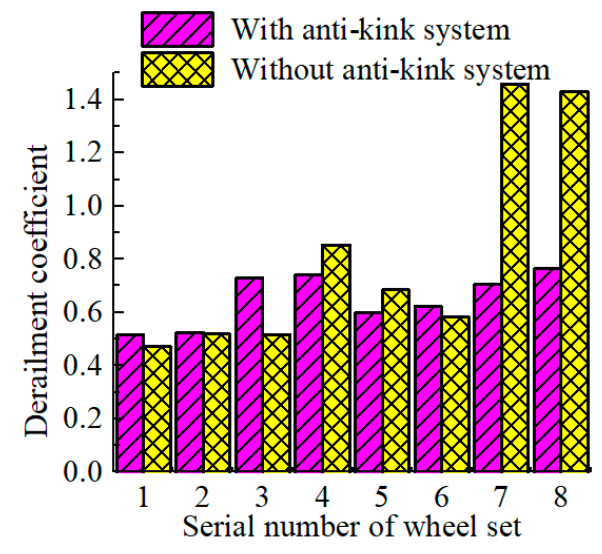

Figure 9. Derailment coefficient.

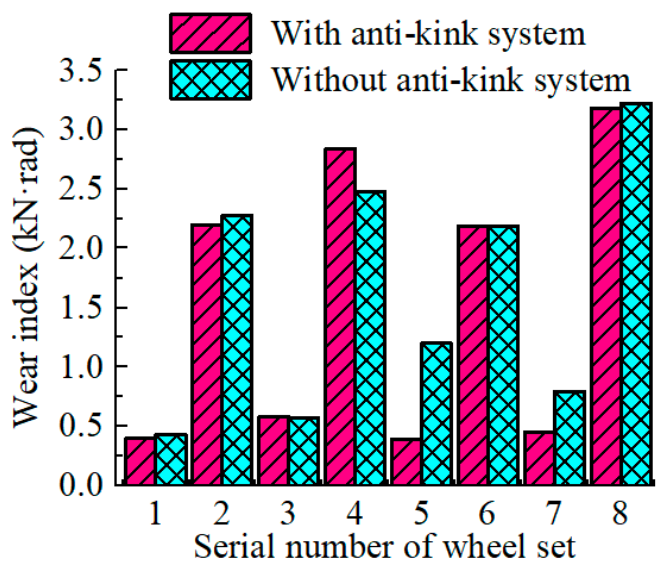

Figure 10. Wear index.

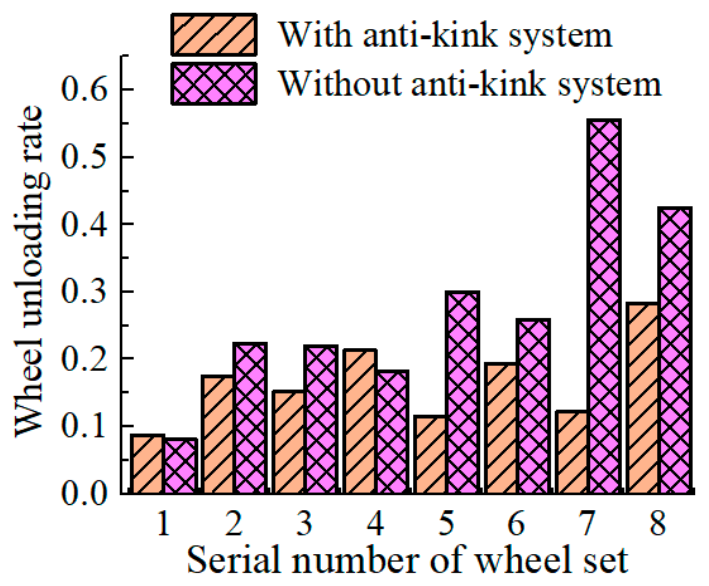

Figure 11. Wheel unloading rate.

Figures 13 and 14 show the change of vehicle body stability index, the maximum lateral stability index of vehicle body without anti-kink system is 2.28 , which is concentrated in M vehicle, the maximum lateral stability index of vehicle body with anti-kink system is 1.74 , which is also concentrated in $\mathrm{M}$ 
vehicle. Because the vehicle $\mathrm{T}$ has no longitudinal coupling system, and the bogie lateral movement is larger than the other three vehicles, the yaw angle of $\mathrm{T}$ vehicle is larger and the anti-kink system force is larger. The maximum vertical stability index of vehicle body without the anti-kink system is 1.74, concentrated in $\mathrm{M}$ vehicle, the maximum vertical stability index of vehicle body with the anti-kink system is 1.26, concentrated in Mc1 vehicle. So the stability of vehicles with anti-kink system are better than those without anti-kink system. The amplitude and frequency of yaw head of vehicle body have influence on the stability index when the vehicle passes through an S-shaped curve.

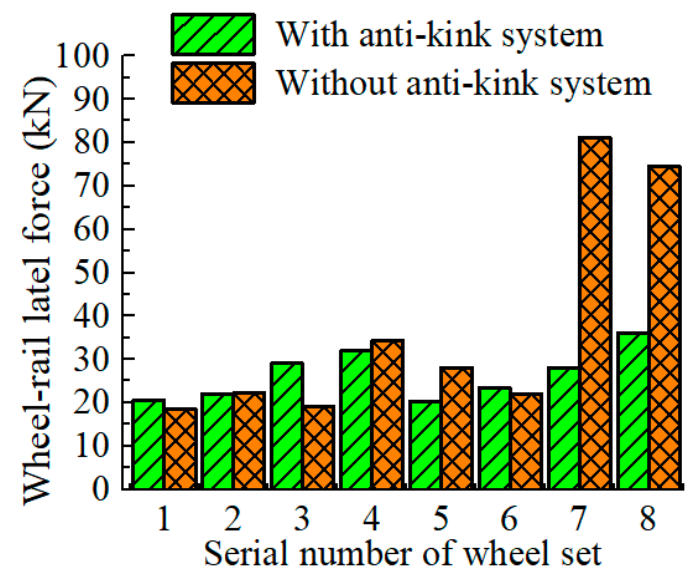

Figure 12. Wheel-rail lateral force.

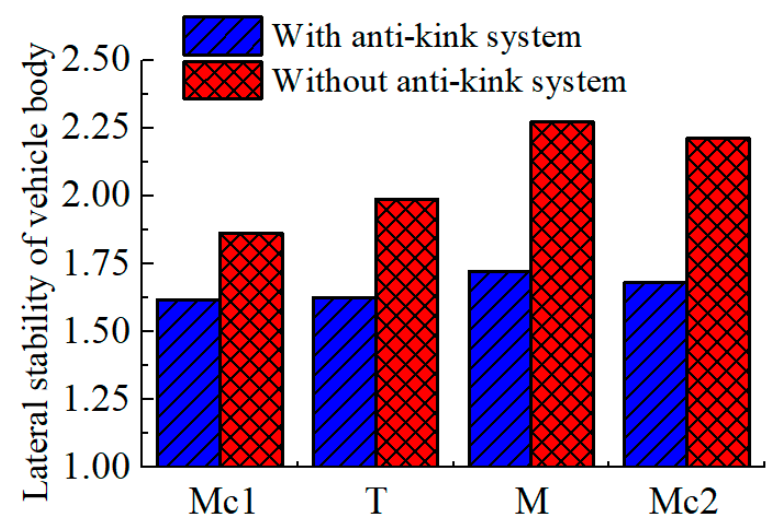

Figure 13. Lateral stability of vehicle body.

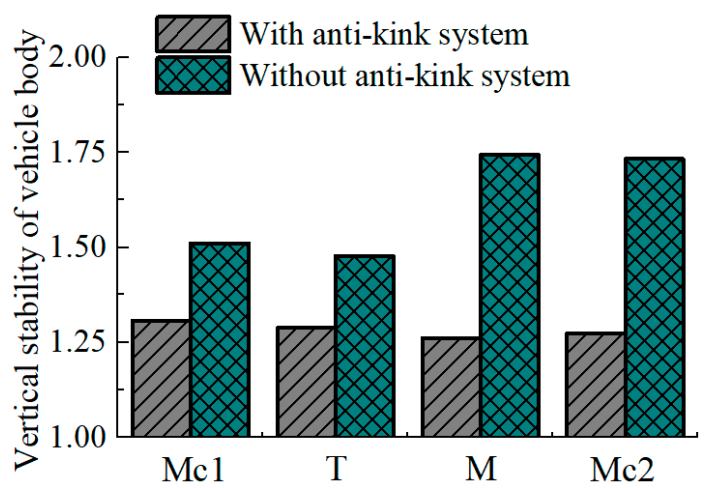

Figure 14. Vertical stability of vehicle body. 


\subsection{C-Shaped Curve Line}

The change of angle between vehicle body and bogie with time for tram running on a C-shaped curve line with the anti-kink system is shown in Figure 15.

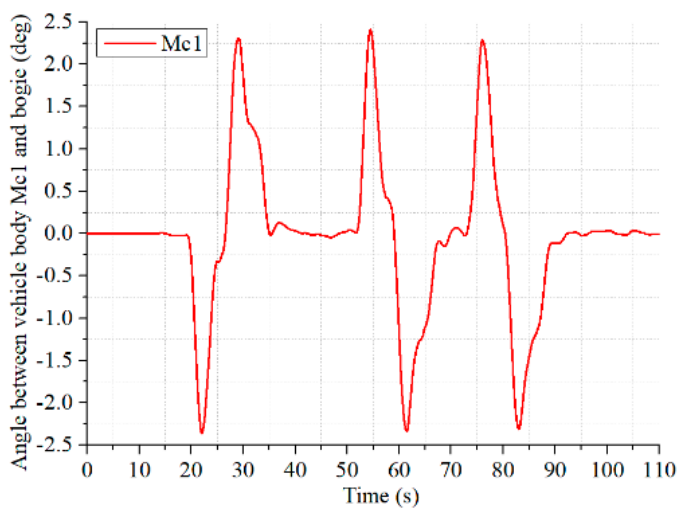

(A) Angle between vehicle body Mc1 and bogie



(C) Angle between vehicle body $\mathrm{M}$ and bogie

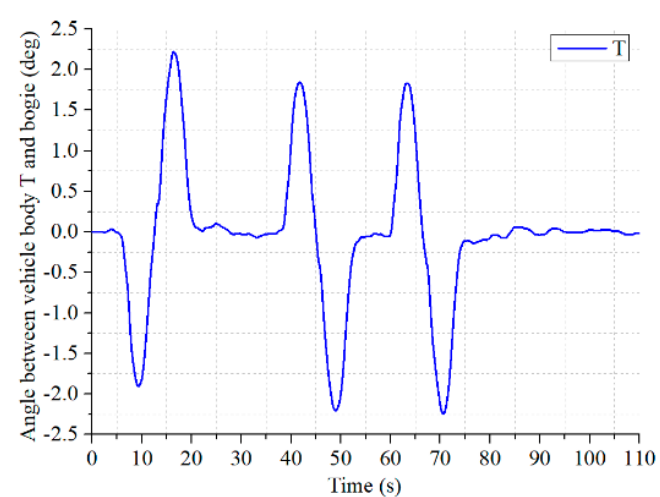

(B) Angle between vehicle body $\mathrm{T}$ and bogie



(D) Angle between vehicle body Mc2 and bogie

Figure 15. Angle between vehicle and bogie with hydraulic anti-kink system.

When the vehicle without the anti-kink system passes through the C-shaped curve, the change trend of the angle between the vehicle body and its bogie is different. The maximum angle between the vehicle body and the bogie can reach $2.39^{\circ}$, which is located in the Mc1 vehicle. Due to the buffer hydraulic cylinder of the working hydraulic anti-kink system, the overall stiffness is relatively small, allowing a certain difference in the angle between the front and rear vehicle bodies and the corresponding bogies, there will be a certain difference between the angles between the vehicle body and the bogie, and there is a delay in the rotation of the front and rear vehicle bodies relative to the corresponding bogies.

Figure 16 shows the change of the maximum derailment coefficient of each wheel group under the operating conditions. When the vehicle passes through the C-shaped curve at a faster speed, the most front-end bogie suffers a greater lateral impact. The maximum derailment coefficient without the anti-kink system reaches 1.48, the maximum derailment coefficient of installing the anti-kink system wheel set is 0.82 . Figure 17 shows the change of wear index. After the anti-kink system is installed, the change range of wear index is small, the wear of front wheel set is large, and the wear of rear wheel set is small. Figure 18 shows the variation of the maximum wheel unloading rate under operating conditions. The maximum wheel unloading rate of the vehicles without the anti-kink system reaches 
0.58 , close to the specified limit, and the maximum wheel unloading rate with the anti-kink system is 0.29 . Figure 19 shows the variation of the maximum wheel-rail lateral force under the operating conditions. The maximum wheel-rail lateral force of the vehicles without the anti-kink system reaches $85.1 \mathrm{KN}$, far exceeding the maximum value allowed by the standard. The maximum wheel-rail transverse force of the vehicles with the anti-kink system is $39.8 \mathrm{KN}$, which is lower than the national standard value of $43 \mathrm{KN}$. Therefore, the anti-kink system can improve the safety of a single tram passing through the C-shaped curve.

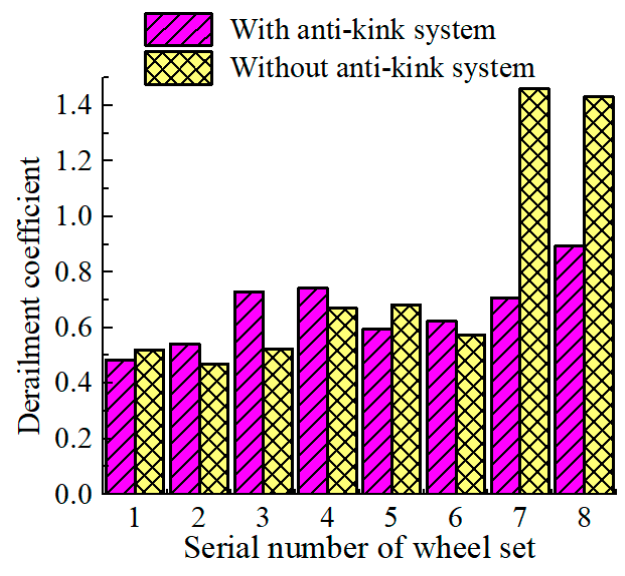

Figure 16. Derailment coefficient.



Figure 17. Wear index.

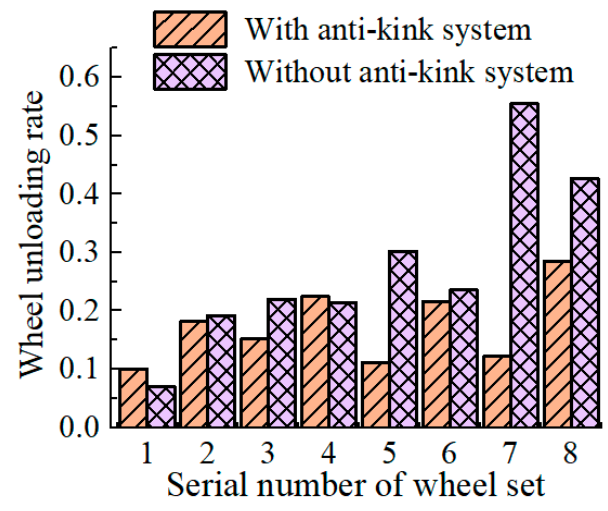

Figure 18. Wheel unloading rate. 


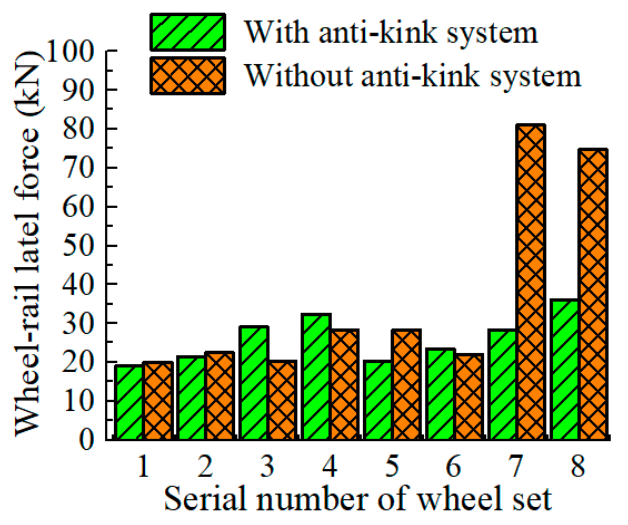

Figure 19. Wheel-rail lateral force.

Figures 20 and 21 show the change of vehicle body stability index and the maximum lateral stability index of vehicle body without the anti-kink system is 2.21 , which is concentrated in $\mathrm{M}$ vehicle, the maximum lateral stability index of vehicle body with the anti-kink system is 1.79 , which is concentrated in Mc2 vehicle. The maximum vertical stability index of vehicle body without the anti-kink system is 1.62, concentrated in $\mathrm{M}$ vehicle, the maximum vertical stability index of vehicle body with the anti-kink system is 1.21 concentrated in Mc2 vehicle. So the stability indexes of vehicles with the anti-kink system are better than those without the anti-kink system. The amplitude and frequency of yaw head of vehicle body have influence on the stability index when the vehicle passes through the C-shaped curve.

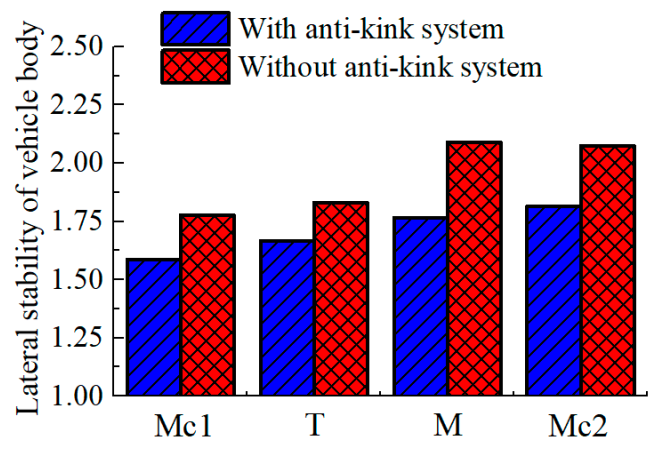

Figure 20. Lateral stability of vehicle body.

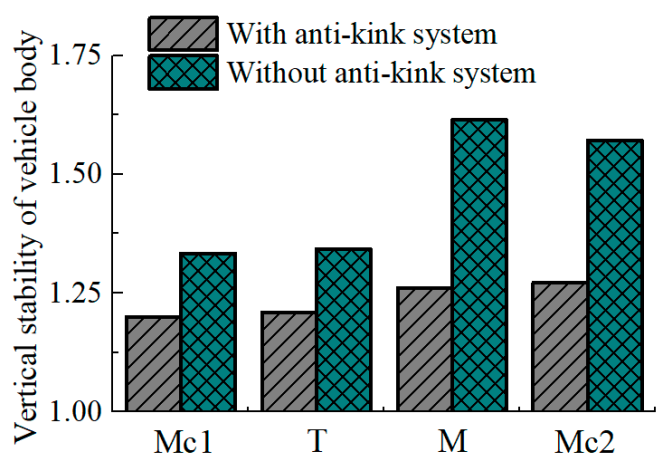

Figure 21. Vertical stability of vehicle body.

\section{Experiment}

The displacement between each bogie and its corresponding vehicle body was measured by two displacement sensors. Which were respectively installed at the edge of the vehicle body and the end 
of the frame to test the lateral displacement of the outermost end of the bogie relative to the vehicle body. The installation diagram is shown in Figure 22. The simplified dimensioning of displacement measurement is shown in Figure 23.

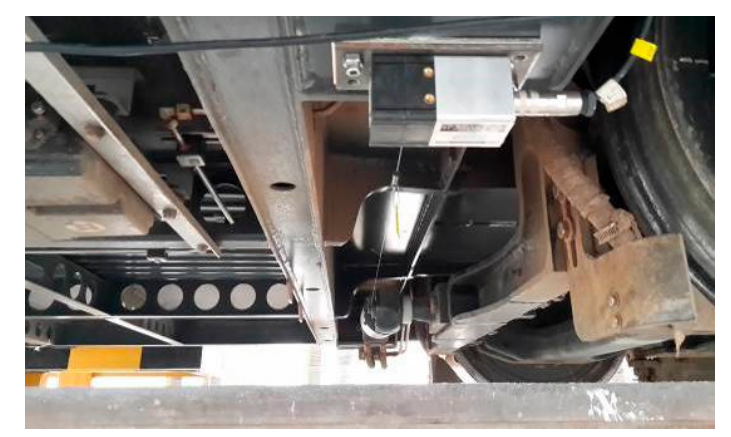

Figure 22. Schematic diagram of sensor installation position.

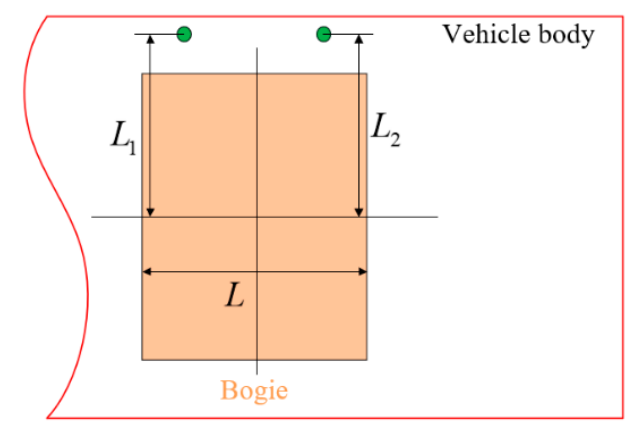

Figure 23. Simplified dimensioning of displacement measurement.

Referring to the Figure 23, the yaw angle between vehicle body and bogie is expressed as:

$$
\gamma_{\text {bogie }}=\frac{180\left(L_{1}-L_{2}\right)}{\pi L}
$$

Here: $L_{1}$ and $L_{2}$ are the horizontal lateral displacement of bogie frame relative to vehicle body and $L$ is the distance between two horizontal measuring points.

The following is the angle change of the low-floor tram Mc1 and T relative to their own bogies on the S-shaped curve line, as shown in Figure 24.

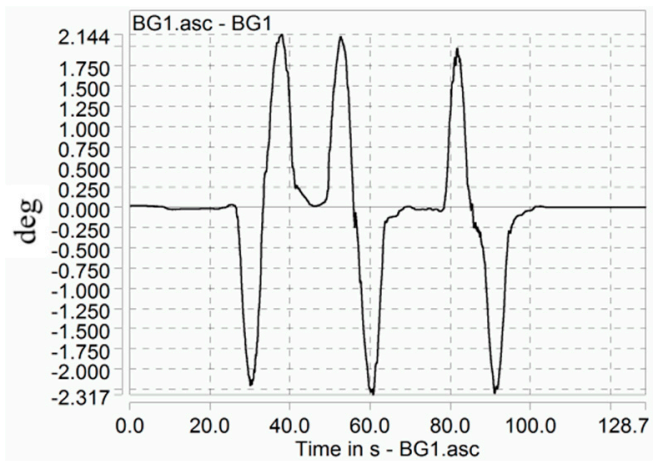

Mc1

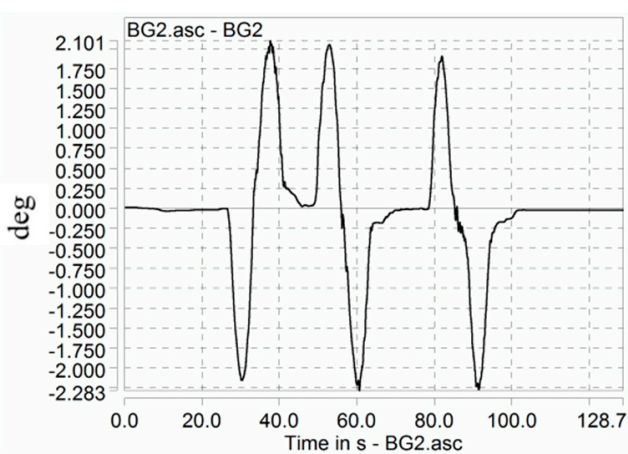

$\mathrm{T}$

Figure 24. Mc1 and T relative to their own bogies on the S-shaped curve line. 
Due to the limitation of experimental conditions, there is no separate $\mathrm{R}=25 \mathrm{~m}$ curve radius curve. Therefore, a continuous curve with $R=25 \mathrm{~m}$ curve radius and $C$ curve is selected, that is, after the vehicle passes through the curve with $R=25 \mathrm{~m}$ small curve radius at the speed limit, and then passes the $C$ curve track at the speed limit. The test results of the $R=25 \mathrm{~m}$ curve and the $C$-shaped curve are displayed together as shown in Figure 25.

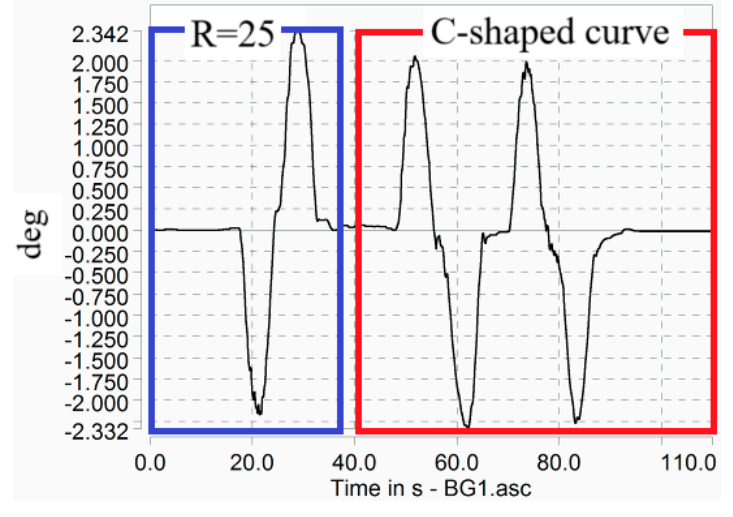

Mc1

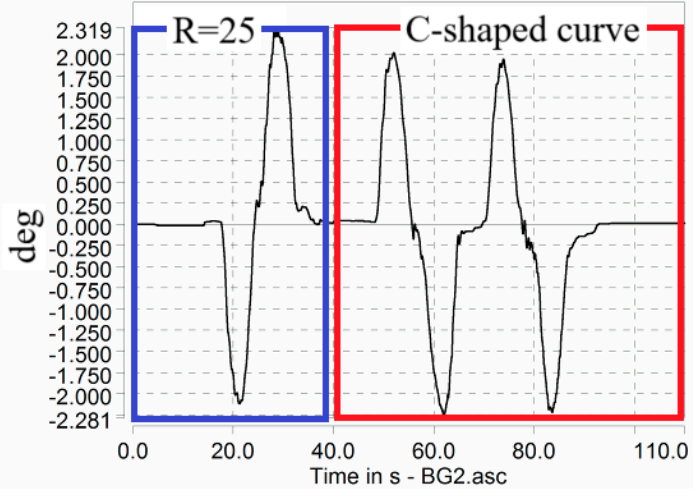

$\mathrm{T}$

Figure 25. Mc1 and T relative to their own bogies on the C-shaped curve line.

\section{Discussion}

Compared with the experimental results (Figure 24) and the simulation results (Figure 8), the curve changes are consistent, but the peak value of the yaw angle is different. The difference between the experimental results and the simulation results (S-shaped curve line) is shown in Table 5.

Table 5. Difference between the experimental results and the simulation results, (S-shaped curve line).

\begin{tabular}{cccccc}
\hline Line Condition & Data Source & Vehicle & Max (deg) & Min (deg) & Error \\
\hline \multirow{3}{*}{ S-shaped curve line } & Simulation & Mc1 & +2.42 & -2.34 & \multirow{2}{*}{$13.1 \% / 1.3 \%$} \\
\cline { 2 - 5 } & Experiment & Mc1 & +2.14 & -2.31 & \\
\cline { 2 - 5 } & Simulation & $\mathrm{T}$ & +1.99 & -2.20 & \multirow{2}{*}{$5.2 \% / 3.5 \%$} \\
\cline { 2 - 5 } & Experiment & $\mathrm{T}$ & +2.10 & -2.28 & \\
\hline
\end{tabular}

Compared with the experimental results (Figure 25) and the simulation results (Figure 15), the curve changes are consistent, but the peak value of the yaw angle is different. The difference between the experimental results and the simulation results is shown in Table 6.

Table 6. Difference between the experimental results and the simulation results, $(\mathrm{R}=25 \mathrm{~m}$ and C-shaped curve line).

\begin{tabular}{|c|c|c|c|c|c|}
\hline Line Condition & Data Source & Vehicle & Max (deg) & Min (deg) & Error \\
\hline \multirow{4}{*}{$\mathrm{R}=25 \mathrm{~m}$} & Simulation & Mc1 & +2.29 & -2.33 & \multirow{2}{*}{$2.1 \% / 3.6 \%$} \\
\hline & Experiment & Mc1 & +2.34 & -2.25 & \\
\hline & Simulation & $\mathrm{T}$ & +2.25 & -1.96 & \multirow{2}{*}{$2.6 \% / 5.8 \%$} \\
\hline & Experiment & $\mathrm{T}$ & +2.31 & -2.08 & \\
\hline \multirow{4}{*}{ C-shaped curve line } & Simulation & Mc1 & +2.39 & -2.32 & \multirow{2}{*}{$16.6 \% / 0.4 \%$} \\
\hline & Experiment & Mc1 & +2.05 & -2.33 & \\
\hline & Simulation & $\mathrm{T}$ & +1.92 & -2.24 & \multirow{2}{*}{$4.5 \% / 1.8 \%$} \\
\hline & Experiment & $\mathrm{T}$ & +2.01 & -2.28 & \\
\hline
\end{tabular}


Referring to Tables 5 and 6, when the vehicles pass the S-shaped curve line and the C-shaped curve line, the maximum yaw angle error of experimental results and simulation results is concentrated on Mc1 vehicle. Because the working conditions of Mc1 as a motor vehicle are much more complicated than that of $\mathrm{T}$ as a trailer when crossing different curves. The reasons for the difference between the experimental results and the simulation results (S-shaped curve and C-shaped curve) may be as follows:

(1) The area of the throttle valve and the size of piston are not very accurate in the hydraulic anti-kink system.

(2) When passing through a small curve, the wheel rail resistance is very large, and the actual running speed cannot be as constant as the simulation environment.

(3) In the actual operation process, $\mathrm{M}, \mathrm{Mc1}$, and $\mathrm{Mc} 2$ vehicles have power, and the drive system has the ability of traction torque control. However, the simulation process relies on the No. 9 hinge to simulate the vehicle operation conditions, which is different from the real situation.

After installing the anti-kink system, the front and rear vehicle bodies have internal relations in parameters. The original secondary suspension parameters and workshop hinge parameters are not necessarily the best parameters after installing the anti-kink system. The secondary suspension parameters and the hinge stiffness between the vehicle bodies can be further optimized, such as the spring stiffness of the buffer hydraulic cylinder or the sectional area of the bypass throttle valve, so as to make the anti-kink system to achieve better performance.

Although the simulation has some defects, the consistency of the change curve shows the accuracy of the model.

Author Contributions: Methodology, all authors; Software, all authors; writing-original draft preparation, X.L., Z.C., and Y.J.; writing-review and editing, X.L., Z.C., Y.J., and J.L.; supervision, Z.C. and J.L.; project administration, X.L. and Y.J. All authors have read and agreed to the published version of the manuscript.

Funding: The work was supported by National Natural Science Foundation of China (No. U1934203) and authors would like to thank the State Key Laboratory of Traction Power for providing office, equipment, and materials to this project.

Conflicts of Interest: The authors declare no conflict of interest.

\section{References}

1. Goodall, R.M.; Kort, W. Mechatornic developments for railway vehicles of the future. Control Eng. Pract. 2002, 10, 887-888. [CrossRef]

2. Hoshi, M.; Ookubo, Y.; Nonaka, H.; Yamaguchi, M.; Arai, T.; Kono, H. First 100\% Domestic Low Floor Tram. Mitsubishi Heavy Ind. Tech. Rev. 2006, 43, 1-2.

3. Hondius, H. Three-stage programme put combino tramsback on track. Railw. Grazette Int. 2005, 161, 615-618.

4. Hondius, H. The development of low-flow trams. J. Adv. Transp. 1993, 27, 79-102. [CrossRef]

5. Uhl, T.; Chudzikiewicz, A. Analytical and experimental investigation of low-floor tram dynamics. Veh. Syst. Dyn. 2002, 37, 702-713. [CrossRef]

6. Zhang, X.; Huang, Y.H. Influence of anti-kink system on curve negotiation performance of low-floor tram. Urban Rail Transit Res. 2018, 21, 111-116.

7. Huang, Y.P.; Ji, Y.J.; Gong, D.; Leng, H.; Ren, L.H. Research on the influence of hydraulic anti-kink system on the curve negotiation performance of trams. J. Mech. Eng. 2019, 55, 162-171.

8. Zhu, W.L.; Wu, M.L.; Tian, C.; Zuo, J.Y. Integrated simulation platform for rail vehicle braking system based on multidisciplinary collaborative analysis. J. Transp. Eng. 2017, 17, 99-110.

9. Li, J.; Zhu, X.L.; Ren, L.H. Analysis of damping characteristics of hydraulic anti-kink system for low-floor light rail vehicles. J. Tongji Univ. 2017, 45, 1044-1049.

10. Wang, Y.Q.; Deng, B.; Yang, F. Design and simulation analysis of anti-kink system based on AMESim. Mach. Tool Hydraul. 2019, 47, 59-61. [CrossRef]

11. Ding, W.S.; Tian, Y.Q.; Xiong, Y.L. Research on damping characteristics of anti-kink hydraulic system of low-floor light rail vehicle. Mach. Tool Hydraul. 2019, 47, 71-74. 
12. Liu, X.; Fang, Y. Simulation of curving performance of rubber tyred suspended rail vehicles. J. Shanghai Univ. Eng. Technol. 2015, 29, 298-302.

13. Wang, K.Y.; Zhai, W.M.; Liu, J.X.; Feng, Q.B.; Cai, C.B. Research on lateral dynamic interaction between speed-up train and curved track. China Railw. Sci. 2005, 06, 40-45.

14. Zboinski, K.; Dusza, M. Bifurcation analysis of 4-axle rail vehicle models in a curved track. Nonlinear Dyn. 2017, 89, 863-885. [CrossRef]

15. Fulks, W.B.; Guenther, R.B.; Roetman, E.L. Equations of Motion and Continuity for Fluid Flow. Acta Mech. 1971, 12, 121-129. [CrossRef]

16. Nadal, M.J. Theorie de stabilit' e des Locomotives, part 2, Mouvement de Lacet. Ann. Mines 1896, 10, $230-232$.

(C) 2020 by the authors. Licensee MDPI, Basel, Switzerland. This article is an open access article distributed under the terms and conditions of the Creative Commons Attribution (CC BY) license (http://creativecommons.org/licenses/by/4.0/). 